\title{
Metabolic Bone Disease in the Bariatric Surgery Patient
}

\author{
Susan E. Williams \\ Department of Internal Medicine, Cleveland Clinic, 9500 Euclid Avenue / G-10, Cleveland, OH 44195-0001, USA \\ Correspondence should be addressed to Susan E. Williams, willias9@ccf.org
}

Received 6 October 2010; Accepted 9 November 2010

Academic Editor: Francesco Saverio Papadia

Copyright (C) 2011 Susan E. Williams. This is an open access article distributed under the Creative Commons Attribution License, which permits unrestricted use, distribution, and reproduction in any medium, provided the original work is properly cited.

\begin{abstract}
Bariatric surgery has proven to be a life-saving measure for some, but for others it has precipitated a plethora of metabolic complications ranging from mild to life-threatening, sometimes to the point of requiring surgical revision. Obesity was previously thought to be bone protective, but this is indeed not the case. Morbidly obese individuals are at risk for metabolic bone disease (MBD) due to chronic vitamin D deficiency, inadequate calcium intake, sedentary lifestyle, chronic dieting, underlying chronic diseases, and the use of certain medications used to treat those diseases. After bariatric surgery, the risk for bone-related problems is even greater, owing to severely restricted intake, malabsorption, poor compliance with prescribed supplements, and dramatic weight loss. Patients presenting for bariatric surgery should be evaluated for MBD and receive appropriate presurgical interventions. Furthermore, every patient who has undergone bariatric surgery should receive meticulous lifetime monitoring, as the risk for developing MBD remains ever present.
\end{abstract}

\section{Introduction}

Although obesity is noted throughout recorded history, the prevalence of obesity rapidly reached pandemic proportions during the second half of the 20th century. Paralleling the pandemic, surgical treatment approaches came into vogue, stemming at least in part from the observation that patients who had undergone gastrectomy experienced significant and durable weight loss. It was not until the early 1970s when the development of metabolic bone disease was linked to gastrointestinal surgeries, most notably following gastrectomy, that rapidly became a well-known cause of osteomalacia. Advances in the field of bariatric surgery have addressed many of the more serious postoperative complications in spite of the untoward consequences of bariatric surgery and dramatic weight loss on skeletal health persist.

\section{Normal Nutrient Absorption: It Is All about the Bones}

Recalling the nutrients essential for bone health and their primary sites of gut absorption helps to illustrate why metabolic bone disease is commonly seen in bariatric surgery patients (Figure 1).
Minerals such as calcium, magnesium, and many trace elements are absorbed predominantly in the proximal small bowel. Calcium absorption is also driven by physiologic need and can be absorbed via active transport throughout the duodenum, the ileum, and, to a lesser degree, the jejunum and colon when need be [1]. Proteins and fats are absorbed in the proximal bowel after the prerequisite actions of pancreatic enzymes. The water-soluble vitamins are absorbed in the proximal small bowel with the exception of $\mathrm{B}_{12}$ which is absorbed principally in the terminal ileum. Vitamin D and the other so-called "fat-soluble" vitamins are mainly absorbed by passive diffusion in the proximal and mid small intestine in a process that is not fat-dependant per se but highly dependant on the presence of bile salts [2-4].

\section{Bariatric Surgery and MBD: A Causal Relationship Established}

Gastrointestinal surgeries resulting in weight loss had their beginnings in the 1940s originally designed to treat, among other maladies, gastric ulcers. By the early 1950's this clinical observation led to the first intestinal bypass surgeries performed expressly for weight reduction. The 


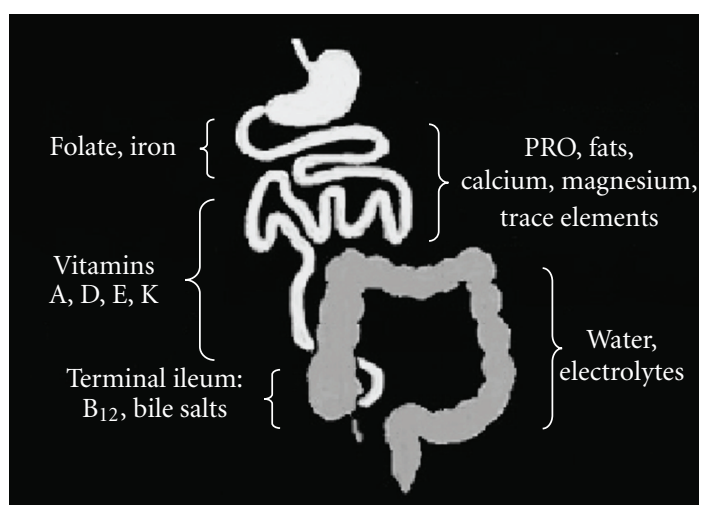

FIGURE 1: Primary sites of absorption of nutrients essential for bone health.

earliest procedures bypassed a great deal more of the small intestine than modern day procedures and did indeed result in dramatic weight loss but were accompanied by severe diarrhea, electrolyte imbalance, hepatic failure, and a high rate of mortality. In the 1960s procedures such as the "144" jejuno-ileal bypass where all by 14 inches of proximal jejunum were bypassed and anastamosed to the terminal ileum (4 inches), promoted significant weight loss with relatively fewer complications but severe malabsorption, protein and vitamin deficiencies, renal oxalate stones, and hepatic failure led to unacceptable complication and mortality rates [5].

The first articles identifying metabolic bone disease following gastrointestinal surgeries were published in the 1970's most notably following gastrectomy and jejuno-ileal bypass [6]. In fact, the prevalence and severity of nephrolithiasis following the 14-4 surgery necessitated surgical reversal in many patients by the fifth postsurgical year, and forced the abandonment of the procedure later that decade.

Since then, publications citing a causal relationship between bariatric surgery and metabolic bone disease number into the multiple of hundreds. The time from surgery to diagnosis ranges from 8 weeks to greater than 32 years, and no bariatric procedure to date has been exempt [7-12]. But undoubtedly the most profound clinical findings have been in older patients who underwent weight loss surgery in the 1970s and were subsequently treated for chronic renal oxalate stones and "severe osteoporosis" when in fact chronic, severe malabsorption, and steatorrhea resulted in profound metabolic derangement, nutrient deficiencies, and osteomalacia $[9,13]$.

Today there are multiple, safe, effective bariatric procedures that are classified by their predominant mechanism of action. Restrictive procedures such as gastric banding and the gastric sleeve promote weight loss by reducing the size of the stomach thereby severely limiting oral intake. Malabsorptive procedures bypass some portion of the small intestine and promote weight loss by decreasing the absorption of caloriecontaining nutrients. And combination procedures, such as the Roux-en-Y gastric bypass and biliopancreatic diversion, limit the size of the stomach as well as bypass part of the small intestine.
Exclusively restrictive procedures, formerly presumed not to alter bone metabolism, appear to place patients at risk of MBD due to inadequate intake of calcium, vitamin $\mathrm{D}$, and protein $[14,15]$. Regardless of the surgical procedure, profound deficiencies can and do occur and as a result, all patients who have undergone a surgical weight loss procedure should undergo routine screenings for metabolic bone disease.

\section{Voluntary Weight Loss: Involuntary Bone Loss}

Voluntary weight loss of approximately $10 \%$ on the part of an obese or overweight individual, whether it is achieved as a result of bariatric surgery or dieting, results in bone loss at all sites of $1-2 \%$ [16-19]. This loss appears to vary among populations in that premenopausal women less than 45 years of age may be able to lose a moderate amount of weight without a significant increase in fracture risk, while a study of overweight men found a $7 \%$ weight loss resulted in a $1 \%$ bone loss [20].

Specifically, weight reduction decreases calcium absorption through several proposed mechanisms, with a subsequent rise in PTH and increase in bone resorption, and the percentage of bone lost as a result of weight loss correlates strongly with the velocity at which the weight is lost. Proposed mechanisms include effects due to increased levels of circulating cortisol, and decreased levels of circulating estrogen, IGF-1, leptin, ghrelin, and GLP-2, particularly in patients who have undergone bariatric surgery [20].

Postsurgically, rapid weight loss of $50 \mathrm{~kg}$ to greater than $100 \mathrm{~kg}$ is not uncommon among successful bariatric patients, and this combined with severely restricted oral intake, decreased calcium absorption, and vitamin D deficiency places these patients at extremely high risk for the rapid development of MBD [10, 21, 22]. One large study noted the development of metabolic bone disease in greater than $70 \%$ of patients having undergone a malabsorptive procedure, a second study detected increased markers of bone resorption as soon as 8 weeks after bariatric surgery, regardless of whether the patient underwent a malabsorptive or restrictive bariatric procedure, and yet another study that examined patients 12 months after undergoing gastric banding found that $48 \%$ had a statistically significant bone mineral reduction of greater than three percent $[7,9,14]$.

Attempts to protect the skeleton and mitigate the activation of the calcium-PTH axis during weight reduction with supplemental calcium and vitamin D have had mixed results. One current hypothesis supports the fact that the usual recommended intake of calcium is inadequate to during weight loss, and higher levels of 1600-1800 mg/day should be recommended, while the required level of supplemental Vitamin D during periods of rapid weight loss remains unclear [23, 24].

Inadequate protein intake also has a detrimental effect on bone and may play a key role in the development of MBD in this population. The intake of lean protein is highly emphasized in the immediate postoperative period as a body 
protein-sparing strategy; however tolerance, compliance, and malabsorption issues frequently result in inadequate intake and frank protein deficiency. This is discussed in further detail below.

\section{Four Paradoxes of Bariatrics and MBD}

As medical professionals, we made assumptions every day regarding our patients, fostered at least in part by the everincreasing speed at which we must see, assess, diagnose, and treat. Although many suppositions are both legitimate and accurate, consider the following commonly made assumptions that are, in fact, fundamental paradoxes when it comes to bariatric patients and the risk for metabolic bone disease.

5.1. Obesity $\neq$ Well-Nourished. The first paradox to consider in approaching the morbidly obese patient is that the presence of obesity does not equate to being well nourished. In fact, body mass index (BMI) quantifies body mass, not nutrition status, and should not be presumed to be a surrogate marker for the nutritional status of the individual. Recognizing this fact allows the clinician to maintain an appropriately high index of suspicion for underlying deficiencies that could serve as clues to the presence of metabolic bone disease.

5.2. Morbid Obesity $\neq$ Better Bone Quality. Morbid obesity has historically been viewed as having a protective effect against the development of osteoporosis. But the second paradox to be attentive to in this population is that although the bone mineral density as measured by dual-energy X-ray absorptiometry (DXA) may be normal, it does not equate to normal (or better) bone quality. In fact, sequestration of vitamin $\mathrm{D}$ in the adipocytes, frank vitamin $\mathrm{D}$ and calcium deficiencies, and secondary hyperparathyroidism (HPTH) are common in extremely obese individuals presenting for bariatric surgery, and all can have a profound effect on bone quality $[8,9,21,25]$. Studies attempting to define the prevalence of vitamin $\mathrm{D}$ deficiency have identified rates in excess of $60 \%$ among patients selected to undergo weight loss surgery $[9,26]$. Similarly, the prevalence of elevated PTH in this population ranges from $25 \%$ to $48 \%[9,27]$.

5.3. Morbid Obesity $\neq$ Central DXA. The third paradox is that bariatric surgery patients are at high risk for bone loss but DXA, the gold standard for bone density measurement, has limited utility in this population. Weight limitations of DXA tables have typically been $250-275$ pounds. Newer and larger machines can accommodate upwards of 450 pounds but this is still insufficient to accommodate many bariatric patients. Forearm DXA imaging remains the only statistically validated option for assessing bone mineral density and fracture risk in patients who exceed the recommended table weight limit, and should be used for presurgical screening and postsurgical surveillance in this population.

The use of serial DXA in patients who have experienced dramatic weight loss is not without controversy. Discussed elsewhere in the literature, it is important to note that there are some studies that have identified statistically significant accuracy errors in serial central DXA measurements in this population [28].

5.4. Abnormal DXA $\neq$ Osteoporosis. Despite the presence of long-standing morbid obesity, many patients will have abnormally low bone mineral density test results. But abnormal DXA results do not always represent primary osteoporosis, and abnormal DXA results should never evoke a "knee-jerk" reflex response on the part of the clinician to diagnose osteoporosis and start a bisphosphonate. In fact, indiscriminate use of bisphosphonates in this patient population can result in life-threatening complicationsmore on this in a moment.

Abnormal DXA in a bariatric surgery patient often represents secondary bone disease due to nutritional deficiencies, and when secondary bone disease is present, it should become the focus of treatment interventions. A clue to the presence of secondary bone disease may be seen in abnormally low Z-scores. Recall that Z-scores for a reference population are matched to age as well as gender. If the bone mineral density has changed only because of normal aging, $\mathrm{Z}$-scores would be expected to be zero, however if the Zscores are significantly low, this should raise the index of suspicion as to the presence of underlying deficiencies.

\section{Case in Point}

The DXA image seen in Figure 2 is the nondominant forearm of a woman who recently presented for evaluation and treatment of hypocalcemia. Her medical history was remarkable for bariatric surgery in 1974, chronic renal stones since 1979, and she had been wheelchair bound for 10 years due to numerous fragility fractures and profound proximal weakness. Biochemical indices were significant for hypocalcemia, undetectable 25 -hydroxyvitamin $\mathrm{D}$, elevated alkaline phosphatase, intact parathyroid hormone five times the upper range of normal and very low urine calcium. The T-scores clearly exceed the World Health Organization criteria for osteoporosis, but the Z-scores in concert with the clinical presentation are even more telling.

Delineating secondary bone disease from low peak bone mass often requires additional clinical data and a skilled specialist, but in this case the grossly abnormal biochemical indices and patient presentation is sufficient to confidently make the correct diagnosis and select the appropriate course of treatment.

\section{Other Clues to the Presence of Metabolic Bone Disease}

Months to years prior to the diagnosis of metabolic bone disease, many patients have nonspecific and vague symptoms that are often incorrectly diagnosed as fibromyalgia, rheumatoid arthritis, polymyalgia rheumatica, Paget disease, or depression [8]. But as in other aspects of clinical medicine, there is still no substitute for a thorough history and physical exam. Additionally, bariatric surgery patients should be 

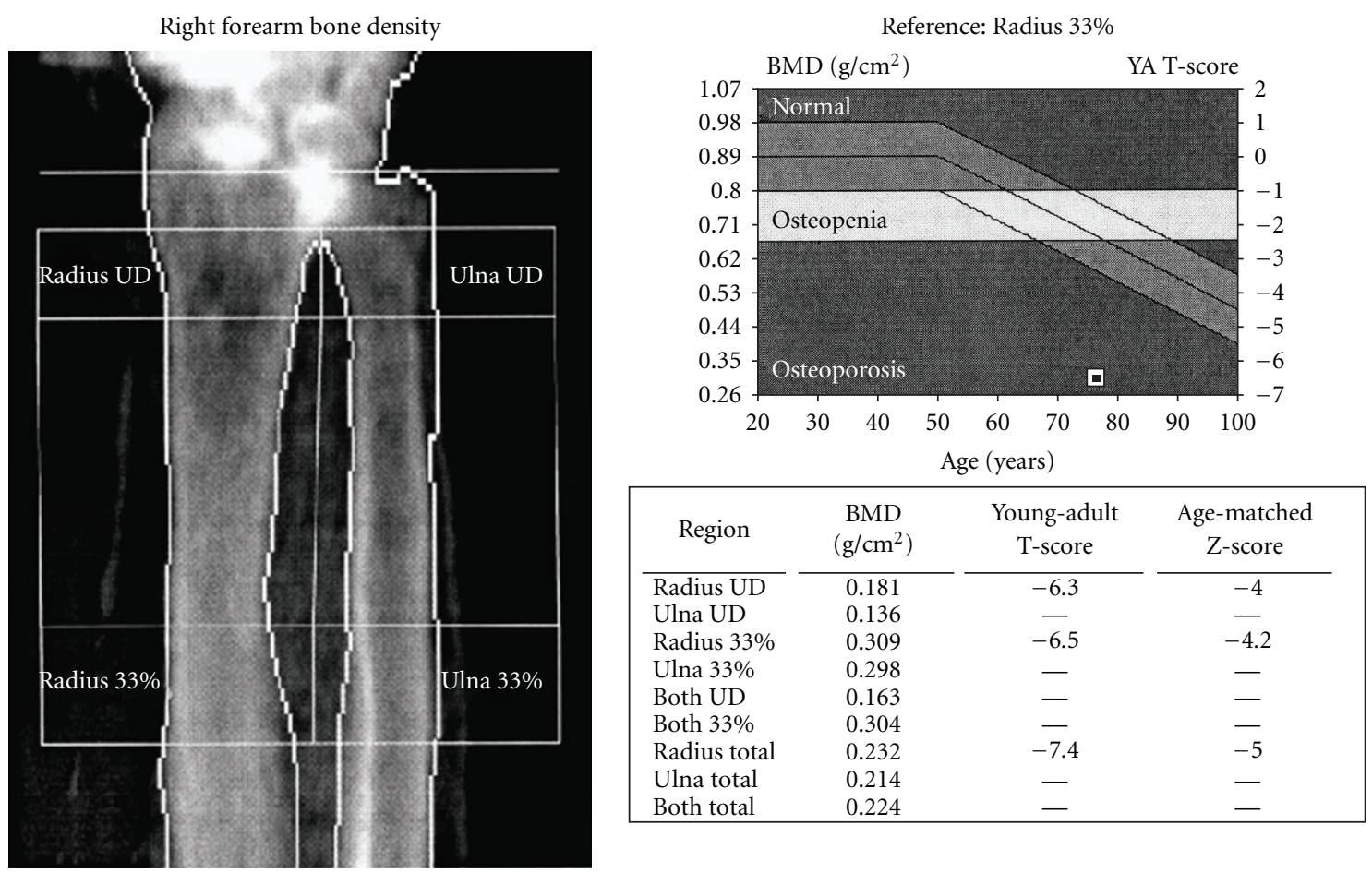

FIGURE 2: DXA-Forearm.

asked about their nutrition practices including protein intake and the use of supplements.

Proximal weakness or complaints of muscle loss; deep, dull, poorly localizing bone pain, and increasing difficulty arising from a chair or ascending a flight of stairs all serve as clues to the possible presence of vitamin $\mathrm{D}$ deficiency and osteomalacia. A history of frequent stooling, steatorrhea, or kidney stones should also heighten clinical suspicion of abnormal calcium metabolism and risk for bone loss.

Finally, undersupplementation in this patient population can rapidly lead to MBD in that maintenance of normal nutrition status and the associated lab parameters typically requires daily protein and supplements in doses that far exceed the current RDAs. It is imperative for clinicians to have a high index of suspicion for the presence of MBD if a patient reports taking only an over-the-counter multivitamin and $500 \mathrm{mg}$ of calcium daily, with little attention paid to the diet.

\section{Calcium Oxalate Stones}

In the intact GI tract, bile acids and free fatty acids are absorbed in the proximal intestine, calcium forms an insoluble precipitate with oxalates, and calcium oxalates are harmlessly excreted in the stool. In the malabsorptive gut, unabsorbed fatty acids bind calcium thereby making it unavailable to bind oxalates, and lingering bile acids and free fatty acids promote increased colonic permeability. The unbound oxalates are readily absorbed in the distal gut resulting in hyperoxaluria thereby increasing the likelihood of oxalate deposition in the renal parenchyma $[29,30]$. One recent study noted a mean time to the development of the first stone after roux-en-y gastric bypass (RNYGB) of 2.9 years with a range of 1 month to 13 years [29].

Although there is evidence that a low-oxalate diet may decrease the risk of stone formation by 20 percent, it is important to keep in mind that dietary oxalates only account for 10-20 percent of total oxalates, with hepatic synthesis accounting for 40-50 percent, and ascorbic acid metabolism accounting for the remaining $40-50$ percent [31]. Oxalates are a ubiquitous component of plants, cereal grains, leafy, and root vegetables, therefore an oxalate-free diet is clearly not possible, and further restriction of the diet following bariatric surgery, with its inherent risks for nutritional inadequacy, is inadvisable. Emphasis on adequate water intake, avoidance of dietary fats and supplemental vitamin $\mathrm{C}$, and supplementation with calcium citrate which helps to increase urinary $\mathrm{pH}$ and retard calcium oxalate crystal formation, have all been demonstrated to be beneficial in preventing calcium oxalate stones in this population [31-33].

\subsection{AACE/TOS/ASMBS Guidelines}

There have been a wide variety of recommendations put forth in the literature for perioperative screening, risk stratification, and management of MBD in bariatric patients [34-37]. With the 2008 publication of the American Association of Clinical Endocrinologists, The Obesity Society, and American Society for Metabolic and Bariatric Surgery (AACE/TOS/ASMBS) medical guidelines for perioperative support of the bariatric surgery patient, there are now evidence-based practice guidelines that include recommendations for testing and management of skeletal and mineral disorders [38]. Although the guidelines serve as an excellent 
general guide, essential information specifically addressing bone disease in this population is still lacking. Specifically, evidence-based guidelines need to be put forth regarding presurgical bone assessment, the preferential use of calcium citrate, the use of cholecalciferol (D3) over $\mathrm{D}_{2}$ or D analogs; the efficacy of UV-B light in patients resistant to oral repletion; the risk for fat-soluble vitamin deficiencies with longterm use of bile acid sequestrants commonly used to treat postoperative diarrhea, and both the risks and questionable efficacy of oral bisphosphonates in this population.

\section{Recommendations}

Presurgically, in addition to the biochemical indices recommended in the AACE/TOS/ASMBS guidelines which include 25-hydroxyvitamin $\mathrm{D}$ and a bone marker of resorption such as urine $n$-telopeptide (NTX), obtain a baseline DXA, even if only the forearm can be imaged because of weight limitations. If the DXA is abnormal, pursue further investigation and treatment as appropriate, with a high index of suspicion for secondary bone disease.

Postsurgically, repeat the DXA in one year, then follow the International Society for Clinical Densitometry (ISCD) guidelines in determining when best to obtain future bone density studies [39].

\section{Calcium Supplementation}

Calcium citrate has been demonstrated to have better bioavailability, superior fractional uptake in bone, and efficacy in normalizing markers of bone turnover when compared to other commercially available calcium supplements [40]. Citrate is metabolized to bicarbonate which has a neutralizing affect on urine, thereby also decreasing the risk of nephrolithiasis.

An optimal daily intake of calcium based on the specific bariatric surgical procedure is currently unknown however attempts to optimize calcium intake is essential, particularly during periods of rapid weight loss [23, 24]. Absorption, separate from the issue of compliance, can be problematic for some patients, therefore judicious monitoring is advised. It is important to remember that calcium homeostasis is a tightly regulated process, maintained by a combination of gut absorption, bone resorption, and renal reabsorption. In the absence of adequate dietary calcium and/or absorption, calcium will be resorbed from bone in order to support calcium-dependant processes; therefore the serum calcium is an unreliable measure of adequate calcium intake. Quantifying urine calcium can assist in assessing the adequacy of calcium intake in that abnormally low urine calcium in the presence of normal renal function suggests inadequate intake and/or absorption. Table 1 provides a summary of current recommendations for this unique population $[13,41]$.

11.1. Vitamin D. Serum 25 -hydroxyvitamin D remains the most accurate marker of vitamin D status, and has wellestablished, strong associations with vitamin D deficiency, fragility fractures, and secondarily elevated parathyroid hormone.
TABLE 1: Recommended daily calcium and vitamin $\mathrm{D}_{3}^{*}$ intake.

\begin{tabular}{lcc}
\hline $\begin{array}{l}\text { Adult women and } \\
\text { men }\end{array}$ & Calcium & Vitamin $\mathrm{D}_{3}$ \\
\hline $\begin{array}{l}\text { During periods of } \\
\text { rapid weight loss }\end{array}$ & $1,500-2000 \mathrm{mg}$ & $1,000 \mathrm{IU}$ \\
$\begin{array}{l}\text { Morbidly obese } \\
\text { patients }\end{array}$ & $1,500 \mathrm{mg}$ & $2,000 \mathrm{IU}$ \\
$\begin{array}{l}\text { Post-Bariatric } \\
\text { surgery patients }\end{array}$ & $1,500-2000 \mathrm{mg}$ & $2,000 \mathrm{IU}-100,000+\mathrm{IU}$ \\
\hline
\end{tabular}

* Doses listed are for maintenance of normal levels. Repletion of vitamin D often requires significantly higher doses.

Correction of vitamin D deficiency in bariatric surgery patients requires more than just an over-the-counter multivitamin. Repletion has been safely achieved by giving 50,000 IU to $100,000 \mathrm{IU}$ cholecalciferol daily for one to two weeks followed by a maintenance dose of 50,000 one to three times weekly. Ergocalciferol as well as the various vitamin $\mathrm{D}$ analogs have not demonstrated the efficacy achieved with cholecalciferol in normalizing blood values or addressing symptoms $[42,43]$.

The absorption of vitamin $\mathrm{D}$ is dependant upon the presence of bile acids. Predictably, it has been demonstrated that taking cholecalciferol with the largest meal of the day promotes improved uptake [44]. And for patients who are unable to achieve normal serum levels with oral supplementation, UV-B phototherapy is an effective alternative $[3,4$, $45,46]$. Having said that, the use of tanning beds, although advocated by few, is not recommended due to the fact that the average tanning bulb emits 95\% UVA and 5\% UVB radiation, imparts a 4 -fold to 15 -fold higher dose of UVA and 2 -fold higher dose of UVB than summer, noontime casual sun exposure, and have been implicated in the development of skin cancers $[47,48]$.

11.2. Cholestyramine. Originally marketed as an effective drug for the reduction of plasma cholesterol because of its ability to sequester intestinal bile acids, cholestyramine is now commonly prescribed for the symptomatic relief of diarrhea. When the drug is used on a short-term basis, there does not seem to be cause for concern however, when prescribed long term as is often the case in bariatric surgery patients, there is an increased risk for alterations in vitamin and mineral metabolism due to the resin's bile acid-binding characteristics [49], and risk of bowel obstruction [50, 51].

Studies conducted in rodents that date back to the 1970s demonstrated a net negative balance for calcium, inadequate vitamin $\mathrm{D}$ absorption, and a subsequent increase in parathyroid hormone secretion due to cholestyramine. Frank osteomalacia was also noted but was found to be reversible with vitamin D supplementation [49]. Subsequent human studies confirmed the relationship between chronic use of bile acid resins, impaired vitamin D absorption, and osteomalacia [52] while at least one longitudinal study refutes the effect on the availability of vitamin $\mathrm{D}$ and the development of secondary hyperparathyroidism [53]. 
The evidence regarding the risk of bowel obstruction due to cholestyramine is more scanty, almost exclusively in the pediatric population, and predominantly in the form of case reports dating back to the late 1960s [54, 55]. The lone exception is a review published in 2007 that while examining safety considerations noted that moderate-to-severe constipation was common, and subsequently compelled the authors to recommend that cholestyramine and other bile acid sequestrants are to "be avoided in patients with recent abdominal surgery and in patients with recent or repeated episodes of intestinal obstruction" [56].

\section{Bisphosphonate Use in Bariatric Surgery Patients}

When a bone loss disease occurs in a bariatric surgery patient, secondary disease should be suspected first, and it is the secondary disease that deserves the primary focus of treatment interventions. The etiology of confirmed vitamin D deficiency, hypocalcemia, elevated alkaline phosphatase, secondary hyperparathyroidism, and accompanying signs and symptoms should be clearly delineated to the degree possible, and appropriate treatment interventions initiated. Abnormal DXA may be indicative of both primary and secondary disease however aggressive treatment of the underlying cause of the secondary disease can result in significant improvements in BMD [41].

Clinical and biochemical resolution of secondary bone disease in the presence of persistently abnormal DXA should prompt treatment considerations for primary bone disease, however concern remains when considering the use of oral bisphosphonates in bariatric surgery patients due to the lack of safely and efficacy data. Specifically, tolerance has not been established in the surgical gut, and risk of ulceration at surgical anastamosis has not been defined. Efficacy of oral bisphosphonates has also come into question following intestinal procedures after which the drug may be malabsorbed. It is for these reasons that if a bisphosphonate is indicated, unless there is evidence to the contrary, intravenous administration is recommended.

\section{Protein and Bone Health}

A systematic review of protein and bone health concluded that diets containing $1.0-1.5 \mathrm{~g} / \mathrm{kg}$ protein are typically optimal for bone health [57]. This is particularly worrisome, in that this suggests the current RDA of $0.8 \mathrm{~g}$ protein $/ \mathrm{kg}$ is insufficient to promote calcium homeostasis. Similarly, the common practice of prescribing 60 to 80 grams of protein after bariatric surgery is often wholly inadequate. It is essential to use an adjusted body weight that approximates metabolically active tissue when calculating protein needs as there is also a link between excessive protein intake, calciuria, and increased fracture risk $[58,59]$.

\section{Bariatric Osteomalacia: An Evolving Concept}

Prior to recent investigations in metabolic bone disease and bariatric surgery, improvement in bone density as a result of vitamin D supplementation had been demonstrated however, the changes were small, of questionable clinical significance, and occurred exclusively in trabecular bone [60].

Bariatric osteomalacia appears to be a unique disorder in that profound improvement in symptoms and bone mineral density measurements have been observed in response to aggressive oral repletion with pharmacologic doses of cholecalciferol and calcium citrate (Figure 3). It has been proposed that these observed responses may be unique to bariatric surgery patients, is a synergistic and graded response of D3 and calcium citrate based on the magnitude of the deficiencies, and that D3 likely has a hormonal effect on cortical bone that is more pronounced in this patient population $[41,61]$. Studies are currently underway in an effort to confirm the observations, characterize the pathophysiology, and define diagnosis, treatment, and preventative guidelines for this increasingly common disorder.

\section{Summary}

Bariatric surgery has proven to be an effective and life-saving measure that provides sustainable weight loss but it is not without risk of complications, to include metabolic bone disease.

There is a causal, multifactorial relationship between bariatric surgery and MBD and for that reason MBD remains an ever-present risk in bariatric surgery patients. Patients presenting for bariatric surgery should be evaluated for MBD and receive appropriate presurgical interventions. Postsurgically, the importance of consuming adequate protein and the correct combination of vitamins and minerals cannot be overstated, remembering that no bariatric surgical procedure is risk-free when it comes to the development of metabolic bone disease.

As clinicians, we cannot assume that our morbidly obese patients are well nourished or that they have normal bone quality. Dual-energy X-ray absorptiometry can be used to help assess bone status in the morbidly obese, however if the DXA table limitations prevent imaging the hips and spine, the nondominant forearm is a validated option for quantifying bone mineral density.

Not all abnormal DXA results represent primary osteoporosis and in fact, in the bariatric population, secondary bone disease is the norm and when the diagnosis has been confirmed, treating the underlying cause of the secondary disease must take precedent. DXA Z-scores, if abnormally low, suggest the presence of secondary MBD, however it is important to remember that secondary disease can be present even in the presence of normal scores. Clues such as proximal weakness, a history of renal oxalate stones, chronic steatorrhea, and undersupplementation should serve to alert the clinician to the possible presence of metabolic bone disease.

In addition to the AACE/TOS/ASMBS guidelines, a baseline and one year postoperative DXAs are recommended. The use of calcium citrate and cholecalciferol (vitamin D3) are the recommended forms of these supplements, and in order to achieve and maintain normal serum levels, very 

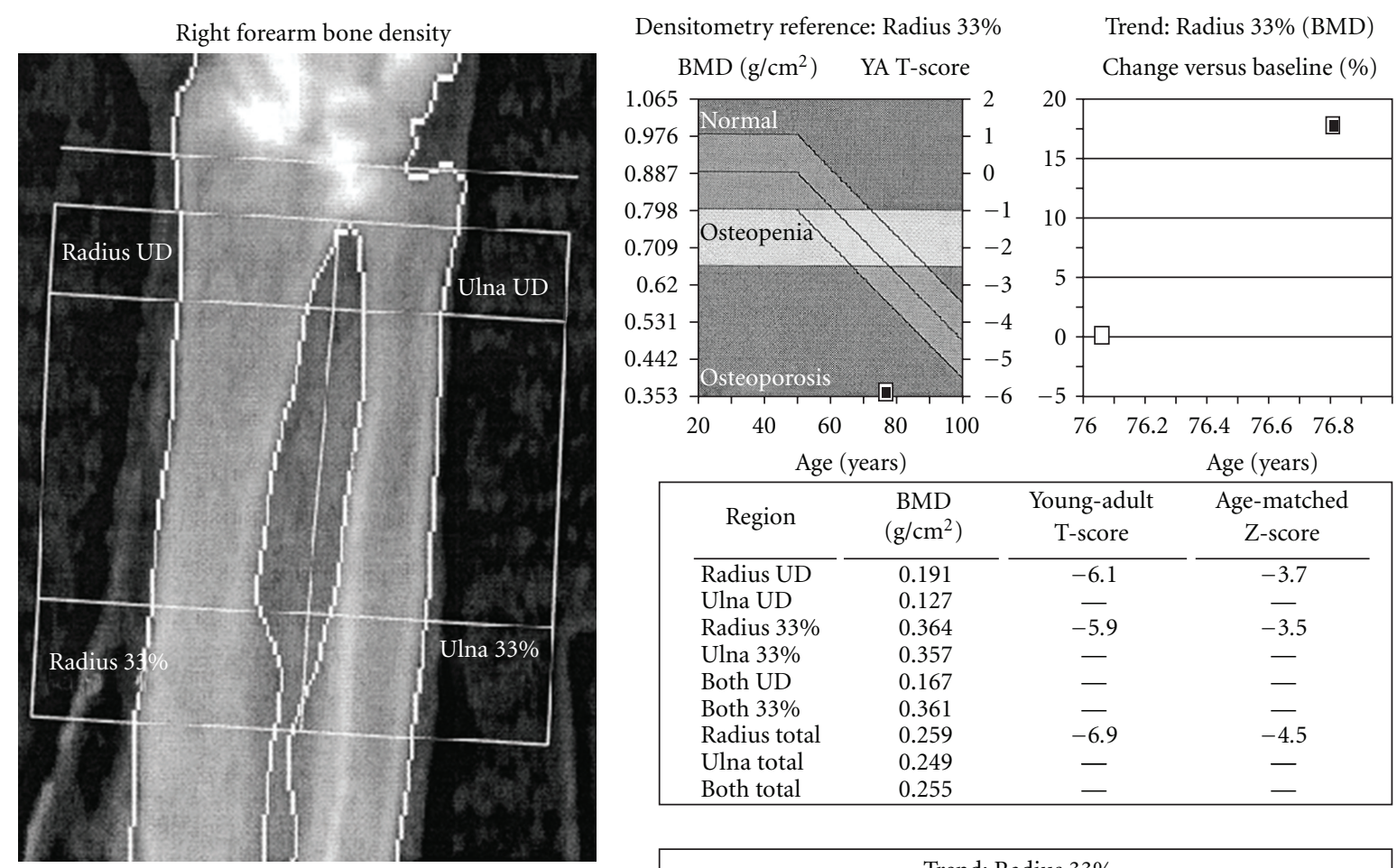

\begin{tabular}{|c|c|c|c|}
\hline Region & $\begin{array}{c}\mathrm{BMD} \\
\left(\mathrm{g} / \mathrm{cm}^{2}\right)\end{array}$ & $\begin{array}{c}\text { Young-adult } \\
\text { T-score }\end{array}$ & $\begin{array}{c}\text { Age-matched } \\
\text { Z-score }\end{array}$ \\
\hline Radius UD & 0.191 & -6.1 & -3.7 \\
\hline Ulna UD & 0.127 & - & - \\
\hline Radius 33\% & 0.364 & -5.9 & -3.5 \\
\hline Ulna 33\% & 0.357 & - & - \\
\hline Both UD & 0.167 & - & - \\
\hline Both 33\% & 0.361 & - & - \\
\hline Radius total & 0.259 & -6.9 & -4.5 \\
\hline Ulna total & 0.249 & - & - \\
\hline Both total & 0.255 & - & 一 \\
\hline
\end{tabular}

\begin{tabular}{|c|c|c|c|c|}
\hline \multicolumn{5}{|c|}{ Trend: Radius 33\% } \\
\hline $\begin{array}{c}\text { Measured } \\
\text { date }\end{array}$ & $\begin{array}{c}\text { Age } \\
\text { (years) }\end{array}$ & $\begin{array}{c}\text { BMD } \\
\left(\mathrm{g} / \mathrm{cm}^{2}\right)\end{array}$ & $\begin{array}{c}\text { Previous } \\
\left(\mathrm{g} / \mathrm{cm}^{2}\right)\end{array}$ & $\begin{array}{c}\text { Previous } \\
(\%)\end{array}$ \\
\hline $11 / 28 / 2007$ & 76.8 & 0.364 & 0.055 & 17.9 \\
\hline $3 / 1 / 2007$ & 76 & 0.309 & - & - \\
\hline
\end{tabular}

Bone mineral density change: $17.9 \%$ increase at the radius $33 \%$ over an 8 -month period Associated clinical changes: decreased bone and muscle pain, no further calcium oxalate stones, improved endurance and strength, ambulating independently

FIGURE 3: Bone remineralization following aggressive oral repletion with cholecalciferol and calcium citrate.

high doses are often required in the bariatric postoperative patient.

Caution is advised when considering the use of certain medications to treat common problems in this patient population. Cholestyramine or other bile acid sequestrants used to control diarrhea in this patient population increase the risk of exacerbating vitamin $\mathrm{D}$ malabsorption and osteomalacia, and may increase the risk of bowel obstruction. The use of bisphosphonates for presumed osteoporosis carries the risk of life-threatening hypocalcemia; efficacy has not been well established in this population, and the risk for ulceration from oral preparations at the surgical anastamosis has yet to be delineated.

Finally, there is emerging evidence that bariatric osteomalacia is a unique and increasingly common phenomenon in bariatric surgery patients that can have a subtle clinical presentation but potentially devastating consequences if left unrecognized. Investigations into the underlying mechanism of the disease, the response to aggressive repletion, and effective preventive strategies are ongoing. The treatment regimen at this point in time includes the use of cholecalciferol and calcium citrate with frequent monitoring and dose adjustments to attain and maintain normal lab parameters.

\section{References}

[1] M. J. Favus and D. Goltzman, "Regulation of calcium and magnesium," in Primer on the Metabolic Bone Diseases and Disorders of Mineral Metabolism, pp. 104-108, American Society for Bone and Mineral Research, Washington, DC, USA, 7th edition, 2008.

[2] D. Shoback, D. Sellmeyer, and D. Bilke, "Metabolic bone disease," in Basic and Clinical Endocrinology, D. G. Gardner and D. Shoback, Eds., pp. 281-345, McGraw-Hill, New York, NY, USA, 8th edition, 2007.

[3] M. F. Holick, "Vitamin D," in Modern Nutrition in Health and Disease, M. E. Shils, J. A. Olsen, M. Shine et al., Eds., pp. 329346, Lippincott Williams \& Wilkins, Philadelphia, Pa, USA, 9th edition, 1999.

[4] C. J. Rosen, "Vitamin D and bone health in adults and the elderly," in Vitamin D: Physiology, Molecular Biology, and Clinical Applications, M. F. Holick, Ed., pp. 287-306, Humana Press, Totowa, NJ, USA, 1999.

[5] K. G. MacDonald, "Overview of the epidemiology of obesity and the early history of procedures to remedy morbid obesity," Archives of Surgery, vol. 138, no. 4, pp. 357-360, 2003.

[6] R. L. Eddy, "Metabolic bone disease after gastrectomy," The American Journal of Medicine, vol. 50, no. 4, pp. 442-449, 1971. 
[7] M. L. Collazo-Clavell, A. Jimenez, S. F. Hodgson, and M. G. Sarr, "Osteomalacia after Roux-en-Y gastric bypass," Endocrine Practice, vol. 10, no. 3, pp. 287-288, 2004.

[8] C. De Prisco and S. N. Levine, "Metabolic bone disease after gastric bypass surgery for obesity," American Journal of the Medical Sciences, vol. 329, no. 2, pp. 57-61, 2005.

[9] S. J. Parikh, M. Edelman, G. I. Uwaifo et al., "Gastric bypass surgery for morbid obesity leads to an increase in bone turnover and a decrease in bone mass," Journal of Clinical Endocrinology \& Metabolism, vol. 89, pp. 1196-1199, 2004.

[10] D. M. Haria, J. D. Sibonga, and H. C. Taylor, "Hypocalcemia, hypovitaminosis D osteopathy, osteopenia, and secondary hyperparathyroidism 32 years after jejunoileal bypass," Endocrine Practice, vol. 11, no. 5, pp. 335-340, 2005.

[11] W. S. Goldner, T. M. O’Dorisio, J. S. Dillon, and E. E. Mason, "Severe metabolic bone disease as a long-term complication of obesity surgery," Obesity Surgery, vol. 12, no. 5, pp. 685-692, 2002.

[12] A. Atreja, C. Abacan, and A. Licata, "A 51-year-old woman with debilitating cramps 12 years after bariatric surgery," Cleveland Clinic Journal of Medicine, vol. 70, no. 5, pp. 417426, 2003.

[13] S. E. Williams and A. A. Licata, "Severe metabolic bone disease in a 76-year-old woman thirty-three years after bariatric surgery," Journal of Clinical Densitometry, vol. 11, no. 3, p. 459, 2008.

[14] N. Pugnale, V. Giusti, M. Suter et al., "Bone metabolism and risk of secondary hyperparathyroidism 12 months after gastric banding in obese pre-menopausal women," International Journal of Obesity, vol. 27, no. 1, pp. 110-116, 2003.

[15] V. Giusti, C. Gasteyger, M. Suter, E. Heraief, R. C. Gaillard, and P. Burckhardt, "Gastric banding induces negative bone remodelling in the absence of secondary hyperparathyroidism: potential role of serum C telopeptides for follow-up," International Journal of Obesity, vol. 29, no. 12, pp. 1429-1435, 2005.

[16] S. A. Shapses, M. Cifuentes, R. Sherrell et al., "Rate of weight loss influences calcium absorption," Journal of Bone and Mineral Research, vol. 17, p. S471, 2002.

[17] S. A. Shapses and C. S. Riedt, "Bone, body weight, and weight reduction: what are the concerns?" Journal of Nutrition, vol. 136, no. 6, pp. 1453-1456, 2006.

[18] D. Chao, M. A. Espeland, D. Farmer et al., "Effect of voluntary weight loss on bone mineral density in older overweight women," Journal of the American Geriatrics Society, vol. 48, no. 7, pp. 753-759, 2000.

[19] P. S. Coates, J. D. Fernstrom, M. H. Fernstrom, P. R. Schauer, and S. L. Greenspan, "Gastric bypass surgery for morbid obesity leads to an increase in bone turnover and a decrease in bone mass," Journal of Clinical Endocrinology and Metabolism, vol. 89, no. 3, pp. 1061-1065, 2004.

[20] S. A. Shapses and M. Cifuentes, "Body weight/composition and weight change: effects on bone health," in Nutrition and Bone Health, M. F. Holick and B. Dawson-Hughes, Eds., pp. 549-573, Humana Press, Totowa, NJ, USA, 2004.

[21] N. Hamoui, G. Anthone, and P. F. Crookes, "Calcium metabolism in the morbidly obese," Obesity Surgery, vol. 14, no. 1, pp. 9-12, 2004.

[22] L. Newbury, K. Dolan, M. Hatzifotis, N. Low, and G. Fielding, "Calcium and vitamin D depletion and elevated parathyroid hormone following biliopancreatic diversion," Obesity Surgery, vol. 13, no. 6, pp. 893-895, 2003.

[23] T. A. Ricci, H. A. Chowdhury, S. B. Heymsfield, T. Stahl, R. N. Pierson, and S. A. Shapses, "Calcium supplementation suppresses bone turnover during weight reduction in postmenopausal women," Journal of Bone and Mineral Research, vol. 13, no. 6, pp. 1045-1050, 1998.

[24] L. B. Jensen, G. Kollerup, F. Quaade, and O. H. Sørensen, "Bone mineral changes in obese women during a moderate weight loss with and without calcium supplementation," Journal of Bone and Mineral Research, vol. 16, no. 1, pp. 141147, 2001.

[25] J. Ybarra, J. Sánchez-Hernández, I. Gich et al., "Unchanged hypovitaminosis D and secondary hyperparathyroidism in morbid obesity after bariatric surgery," Obesity Surgery, vol. 15, no. 3, pp. 330-335, 2005.

[26] N. Puzziferri, J. Blankenship, and B. M. Wolfe, "Surgical treatment of obesity," Endocrine, vol. 29, no. 1, pp. 11-19, 2006.

[27] A. M. Carlin, D. S. Rao, A. M. Meslemani et al., "Prevalence of vitamin D depletion among morbidly obese patients seeking gastric bypass surgery," Surgery for Obesity and Related Diseases, vol. 2, no. 2, pp. 98-103, 2006.

[28] G. M. Blake and I. Fogelman, "How important are BMD accuracy errors for the clinical interpretation of DXA scans?" Journal of Bone and Mineral Research, vol. 23, no. 4, pp. 457462, 2008 .

[29] M. K. Sinha, M. L. Collazo-Clavell, A. Rule et al., "Hyperoxaluric nephrolithiasis is a complication of Roux-en-Y gastric bypass surgery," Kidney International, vol. 72, no. 1, pp. 100107, 2007.

[30] B. G. Duffey, R. N. Pedro, A. Makhlouf et al., "Roux-en$\mathrm{Y}$ gastric bypass is associated with early increased risk for development of calcium oxalate nephrolithiasis," Journal of the American College of Surgeons, vol. 206, no. 6, pp. 1145-1153, 2008.

[31] R. P. Holmes, H. O. Goodman, and D. G. Assimos, "Contribution of dietary oxalate to urinary oxalate excretion," Kidney International, vol. 59, no. 1, pp. 270-276, 2001.

[32] L. K. Massey, M. Liebman, and S. A. Kynast-Gales, "Ascorbate increases human oxaluria and kidney stone risk," Journal of Nutrition, vol. 135, no. 7, pp. 1673-1677, 2005.

[33] E. N. Taylor, M. J. Stampfer, and G. C. Curhan, "Obesity, weight gain, and the risk of kidney stones," Journal of the American Medical Association, vol. 293, no. 4, pp. 455-462, 2005.

[34] M. E. Mason, H. Jalagani, and A. I. Vinik, "Metabolic complications of bariatric surgery: diagnosis and management issues," Gastroenterology Clinics of North America, vol. 34, no. 1, pp. 25-33, 2005.

[35] D. D. Hensrud and M. M. McMahon, "Bariatric surgery in adults with extreme (not morbid) obesity," Mayo Clinic Proceedings, vol. 81, supplement 10, pp. S3-S4, 2006.

[36] B. P. McGlinch, F. G. Que, J. L. Nelson, D. M. Wrobleski, J. E. Grant, and M. L. Collazo-Clavell, "Perioperative care of patients undergoing bariatric surgery," Mayo Clinic Proceedings, vol. 81, no. 10, supplement, pp. S25-S33, 2006.

[37] S. A. Brethauer, B. Chand, and P. R. Schauer, "Risks and benefits of bariatric surgery: current evidence," Cleveland Clinic Journal of Medicine, vol. 73, no. 11, pp. 993-1007, 2006.

[38] J. I. Mechanick, R. F. Kushner, H. J. Sugerman et al., "American Association of Clinical Endocrinologists, The Obesity Society, and American Society for Metabolic \& Bariatric Surgery Medical guidelines for clinical practice for the perioperative nutritional, metabolic, and nonsurgical support of the bariatric surgery patient," Surgery for Obesity and Related Diseases, vol. 4, supplement 1, pp. 1-83, 2008. 
[39] S. Baim, N. Binkley, J. P. Bilezikian et al., "Official positions of the International Society for Clinical Densitometry and Executive Summary of the 2007 ISCD position development conference," Journal of Clinical Densitometry, vol. 11, no. 1, pp. 75-91, 2008.

[40] J. A. Harvey, P. Kenny, J. Poindexter, and C. Y. C. Pak, "Superior calcium absorption from calcium citrate than calcium carbonate using external forearm counting," Journal of the American College of Nutrition, vol. 9, no. 6, pp. 583-587, 1990.

[41] S. E. Williams and A. A. Licata, "Improved forearm bone mineral density after treatment with vitamin $\mathrm{D}_{3}$ in a patient 30 years after bariatric surgery," Journal of Bone and Mineral Research, vol. 23, p. S226, 2008.

[42] L. A. Houghton and R. Vieth, "The case against ergocalciferol (vitamin $\mathrm{D}_{2}$ ) as a vitamin supplement," American Journal of Clinical Nutrition, vol. 84, no. 4, pp. 694-697, 2006.

[43] P. Lips, "Vitamin D deficiency and secondary hyperparathyroidism in the elderly: consequences for bone loss and fractures and therapeutic implications," Endocrine Reviews, vol. 22, no. 4, pp. 477-501, 2001.

[44] G. B. Mulligan and A. Licata, "Taking vitamin D with the largest meal improves absorption and results in higher serum levels of 25-hydroxyvitamin D," Journal of Bone and Mineral Research, vol. 25, no. 4, pp. 928-930, 2010.

[45] V. G. M. Chel, M. E. Ooms, C. Popp-Snijders et al., "Ultraviolet irradiation corrects vitamin D deficiency and suppresses secondary hyperparathyroidism in the elderly," Journal of Bone and Mineral Research, vol. 13, no. 8, pp. 1238-1242, 1998.

[46] M. F. Holick and M. Garabedian, "Vitamin D: photobiology, metabolism, mechanism of action, and clinical applications," in Primer on the Metabolic Bone Diseases and Disorders of Mineral Metabolism, pp. 106-114, American Society for Bone and Mineral Research, Washington, DC, USA, 6th edition, 2006.

[47] J. A. Levine, M. Sorace, J. Spencer, and D. M. Siegel, "The indoor UV tanning industry: a review of skin cancer risk, health benefit claims, and regulation," Journal of the American Academy of Dermatology, vol. 53, no. 6, pp. 1038-1044, 2005.

[48] H. W. Lim, B. A. Gilchrest, K. D. Cooper et al., "Sunlight, tanning booths, and vitamin D," Journal of the American Academy of Dermatology, vol. 52, no. 5, pp. 868-876, 2005.

[49] D. W. Watkins, R. Khalafi, M. M. Cassidy, and G. V. Vahouny, "Alterations in calcium, magnesium, iron, and zinc metabolism in dietary cholestyramine," Digestive Diseases and Sciences, vol. 30, no. 5, pp. 477-482, 1985.

[50] J. A. M. O'Malley, J. T. Ferrucci, and J. T. Goodgame, "Medication bezoar: intestinal obstruction by an Isocal bezoar. Case report and review of the literature," Gastrointestinal Radiology, vol. 6, no. 2, pp. 141-144, 1981.

[51] D. F. Merten and H. Grossman, "Intestinal obstruction associated with cholestyramine therapy," American Journal of Roentgenology, vol. 134, no. 4, pp. 827-828, 1980.

[52] L. C. Knodel and R. L. Talbert, "Adverse effects of hypolipidaemic drugs," Medical Toxicology and Adverse Drug Experience, vol. 2, no. 1, pp. 10-32, 1987.

[53] B. J. Hoogwerf, D. M. Hibbard, and D. B. Hunninghake, "Effects of long-term cholestyramine administration on vitamin $\mathrm{D}$ and parathormone levels in middle-aged men with hypercholesterolemia," Journal of Laboratory and Clinical Medicine, vol. 119, no. 4, pp. 407-411, 1992.

[54] M. I. Cohen, P. R. Winslow, and S. J. Boley, "Intestinal obstruction associated with cholestyramine therapy," New
England Journal of Medicine, vol. 280, no. 23, pp. 1285-1286, 1969.

[55] J. R. Poley, "Cholestyramine and intestinal obstruction," Pediatrics, vol. 61, no. 2, p. 332, 1978.

[56] T. A. Jacobson, A. Armani, J. M. McKenney, and J. R. Guyton, "Safety considerations with gastrointestinally active lipid-lowering drugs," American Journal of Cardiology, vol. 99, supplement 6, pp. 47C-55C, 2007.

[57] J. Z. Ilich and J. E. Kerstetter, "Nutrition in bone health revisited: a story beyond calcium," Journal of the American College of Nutrition, vol. 19, no. 6, pp. 715-737, 2000.

[58] J. Krenitsky, "Adjusted body weight, pro: evidence to support the use of adjusted body weight in calculating calorie requirements," Nutrition in Clinical Practice, vol. 20, no. 4, pp. 468473, 2005.

[59] R. G. Munger, J. R. Cerhan, and B. C. H. Chiu, "Prospective study of dietary protein intake and risk of hip fracture in postmenopausal women," American Journal of Clinical Nutrition, vol. 69, no. 1, pp. 147-152, 1999.

[60] R. Bhambri, V. Naik, N. Malhotra et al., "Changes in bone mineral density following treatment of osteomalacia," Journal of Clinical Densitometry, vol. 9, no. 1, pp. 120-127, 2006.

[61] J. J. Carey, S. E. Williams, B. Richmond, A. A. Licata, and M. F. Delaney, "Extreme bone mineral density increases with vitamin D: an overlooked anabolic therapy? Case series," unpublished. 


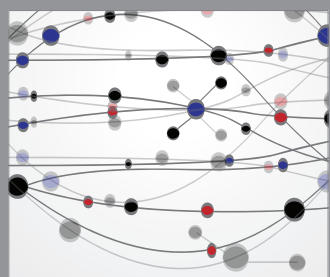

The Scientific World Journal
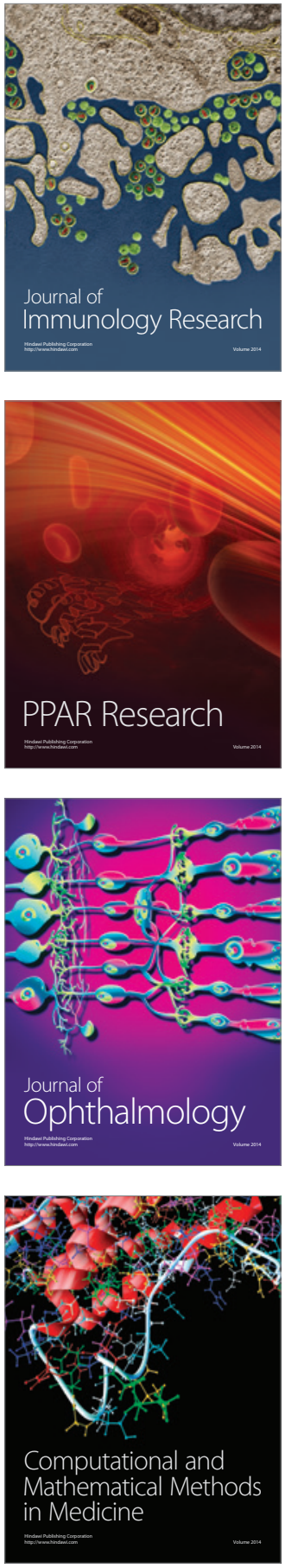

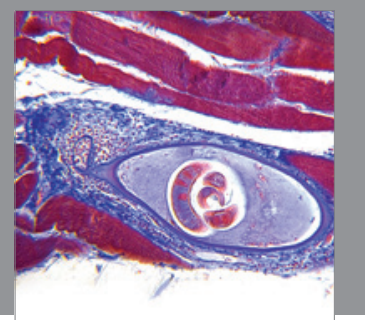

Gastroenterology

Research and Practice
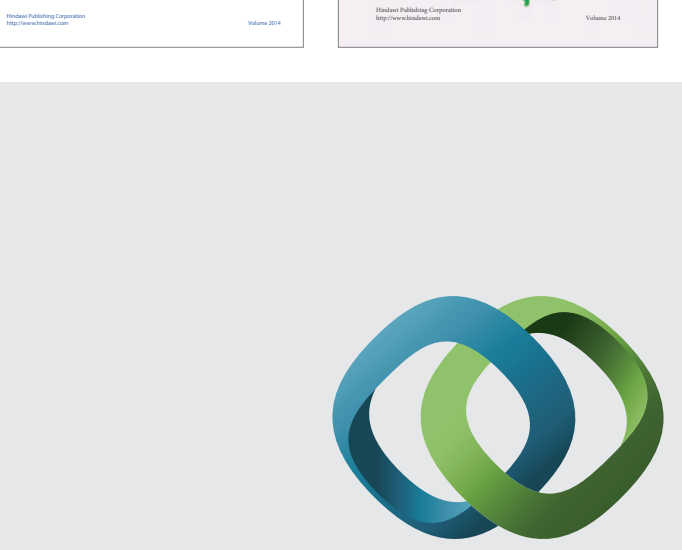

\section{Hindawi}

Submit your manuscripts at

http://www.hindawi.com
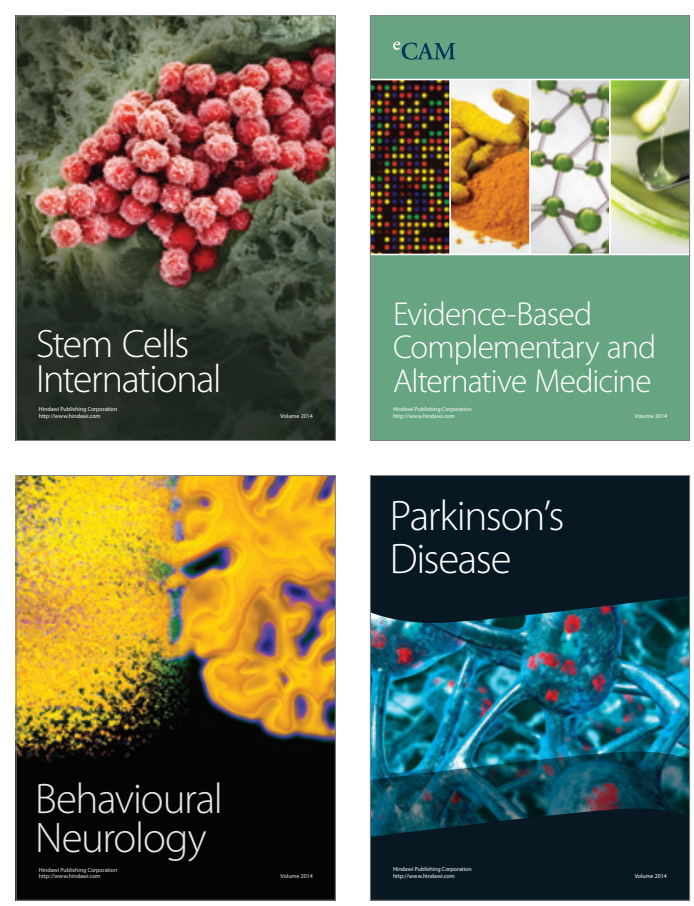

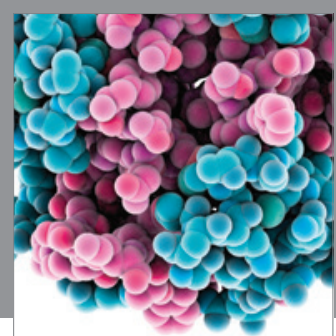

Journal of
Diabetes Research

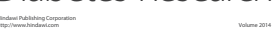

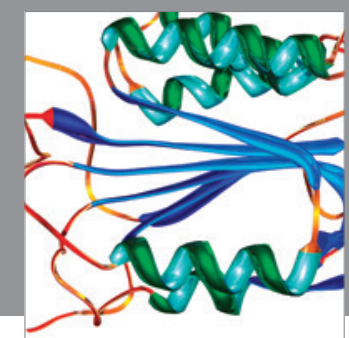

Disease Markers
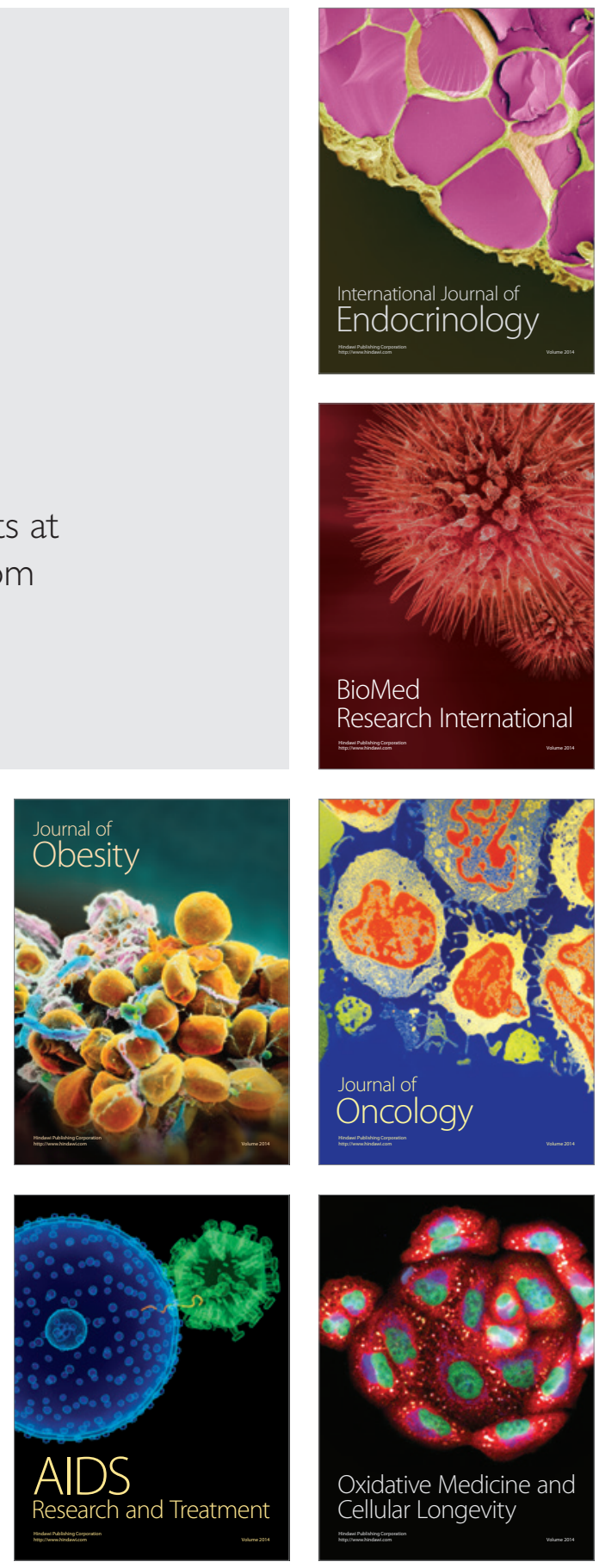